\title{
Male and female differential reproductive rate could explain parental transmission asymmetry of mutation origin in Hirschsprung disease
}

\author{
Anne-Sophie Jannot ${ }^{1,2}$, Jeanne Amiel ${ }^{1,2}$, Anna Pelet ${ }^{1,2}$, Francesca Lantieri ${ }^{3,4}$, Raquel M Fernandez ${ }^{5,6}$, \\ Joke BGM Verheij $^{7}$, Merce Garcia-Barcelo ${ }^{8}$, Stacey Arnold ${ }^{9}$, Isabella Ceccherini ${ }^{4}$, Salud Borrego ${ }^{5,6}$, \\ Robert MW Hofstra ${ }^{10}$, Paul KH Tam ${ }^{8}$, Arnold Munnich ${ }^{1,2}$, Aravinda Chakravarti ${ }^{9}$, Françoise Clerget- \\ Darpoux $^{1,2}$ and Stanislas Lyonnet ${ }^{\star, 1,2}$
}

\begin{abstract}
Hirschsprung disease (HSCR, aganglionic megacolon) is a complex and heterogeneous disease with an incidence of 1 in 5000 live births. Despite the multifactorial determination of HSCR in the vast majority of cases, there is a monogenic subgroup for which private rare RET coding sequence mutations with high penetrance are found ( $45 \%$ of HSCR familial cases). An asymmetrical parental origin is observed for RET coding sequence mutations with a higher maternal inheritance. A parent-oforigin effect is usually assumed. Here we show that a differential reproductive rate for males and females also leads to an asymmetrical parental origin, which was never considered as a possible explanation till now. In the case of HSCR, we show a positive association between penetrance of the mutation and parental transmission asymmetry: no parental transmission asymmetry is observed in sporadic RET CDS mutation carrier cases for which penetrance of the mutation is low, whereas a parental transmission asymmetry is observed in affected sib-pairs for which penetrance of the mutation is higher. This allows us to conclude that the explanation for this parental asymmetry is that more severe mutations have resulted in a differential
\end{abstract} reproductive rate between male and female carriers.

European Journal of Human Genetics (2012) 20, 917-920; doi:10.1038/ejhg.2012.35; published online 7 March 2012

Keywords: Hirschsprung disease; parent-of-origin effect; parental transmission asymmetry; reproductive rate

\section{INTRODUCTION}

Hirschsprung disease (HSCR, aganglionic megacolon) is the most frequent genetic cause of congenital intestinal obstruction. The RET gene, coding for a tyrosine kinase receptor, is implicated in all HSCR cases, but two subgroups of patients have to be distinguished. A small subgroup of patients present a rare coding sequence mutation (CDS) of the RET gene with a high and sex-dependent penetrance. ${ }^{1,2}$ The remaining group of patients have a risk allele for a noncoding polymorphism in an enhancer element of IVS1, rs2435357, with a low and sex-dependent penetrance ${ }^{3-6}$ (found in $90 \%$ of the patient alleles versus $19.4 \%$ in the 1000 genomes project). The subgroup with RET CDS mutations represents $\sim 45 \%$ of familial cases and $7-20 \%$ of sporadic cases. ${ }^{4}$

Bolk et $a l^{1}$ observed that the shared allele at RET among affected sib-pairs is more frequently inherited from the mother than the father. As families with and without RET CDS mutation were pooled, the question of whether parental transmission asymmetry is observed in both types of families remains unanswered. As 35\% of families studied in Bolk et al paper carry a RET CDS mutation, we hypothesized that the parental transmission asymmetry observed in this sample could be the consequence of a high rate of RET CDS mutations only. As rs2435357 was not yet identified as a risk variant at the time of the study in the sample reported in the paper of Bolk et al, ${ }^{1}$ we had to check this hypothesis in another sample. We thus analyzed the data set from the International Hirschsprung Consortium that had been characterized for both RET CDS mutations and rs2435357. This sample includes some of the families previously studied in Bolk et al. ${ }^{1}$ Here, 490 patients were checked for parental origin of rs2435357 risk allele and there was no statistical evidence for preferential paternal or maternal transmission (242 paternal versus 249 maternal transmissions). However, for the 39 patients with an inherited RET CDS mutation and for whom parental origin was available, a parental transmission asymmetry was observed, with 14 mutations paternally inherited and 25 maternally inherited. We therefore focused in the present paper on the subgroup of patients with RET CDS mutations to question the observed parental transmission asymmetry found for RET CDS mutations.

${ }^{1}$ INSERM U-781, AP-HP Hôpital Necker-Enfants Malades, Paris, France; 2Département de Génétique, Université Paris Descartes, Faculté de Médecine, Paris, France; ${ }^{3}$ Department of Health Science, Biostatistics Unit, University of Genova, Genova, Italy; ${ }^{4}$ Laboratorio di Genetica Molecolare, Istituto Gaslini, Genova, Italy; ${ }^{5}$ Unidad de Gestión Clínica de Genética, Reproducción y Medicina Fetal, Instituto de Biomedicina de Sevilla (IBIS), Hospital Universitario Virgen del Rocío/CSIC/Universidad de Sevilla, Sevilla, Spain; ${ }^{6}$ CIBER de Enfermedades Raras (CIBERER), ISCIII, Sevilla, Spain; 7 Department of Genetics, University Medical Center Groningen, University Groningen, Groningen, The Netherlands; ${ }^{8}$ Department of Surgery, The University of Hong Kong, Pokfulam, Hong Kong; ${ }^{9}$ Center for Complex Disease Genomics, McKusick-Nathans Institute of Genetic Medicine, Johns Hopkins University School of Medicine, Baltimore, MD, USA; ${ }^{10}$ Department of Clinical Genetics, Erasmus MC, University of Rotterdam, Rotterdam, The Netherlands

*Correspondence: Professor S Lyonnet, Department of Genetics and INSERM UMR-781, Hôpital Necker-Enfants Malades, 149, rue de Sèvres, 785743 Paris Cedex 15, France. Tel: +33 1444951 36; Fax: +33 1444951 50; E-mail: stanislas.lyonnet@inserm.fr

Received 8 July 2011; revised 17 January 2012; accepted 31 January 2012; published online 7 March 2012 
Parent-of-origin effect, defined by the manifestation of the disease in the offspring depending on the sex of the transmitting parent, is the usual explanation for an observed parental transmission asymmetry. However, a differential reproductive rate among males and females carrying a RET CDS mutation is also an explanation to consider for Hirschsprung disease. Sex ratio in HSCR is indeed unbalanced with more affected males (in our sample, 14 females for 25 males). As RET gene is autosomal, the same number of females and males carry the mutation at conception. Therefore, penetrance differs for male and female mutations carriers. Males carrying a RET CDS mutation are more likely to be affected. Consequently, because of the poor prognosis of the disease until recently, males are less likely to reproduce than females carrying a RET CDS mutation. Thus, although the same proportion of males and females carry a RET CDS mutation at conception, more females than males would be observed among reproducing adults carrying a RET CDS mutation. At the next generation, we would therefore observe more RET CDS mutation inherited from the mother than from the father. This would result in an asymmetrical parental transmission origin.

A differential reproductive rate for males and females due to high and sex-dependent penetrance of a mutation has never been considered in the literature as a possible explanation for a parental transmission asymmetry. Many other ascertainments and mechanisms explaining an asymmetrical parental origin have largely been discussed. ${ }^{7-10}$ However, this literature deals with parental transmission asymmetry observed for common variant with low penetrance, and a differential reproductive rate for males and females cannot be an explanation in these situations.

Parental transmission asymmetry for RET CDS mutations in HSCR cannot be discussed without considering the mutation spectrum observed as private mutation is the rule. These mutations have indeed a large penetrance spectrum. In the case of a mutation with low penetrance, most RET CDS mutation carriers would not be affected and therefore would reproduce. On the contrary, for mutation with near-complete penetrance, as the disease sex ratio is unbalanced, more healthy females than males would be observed, more females than males would reproduce, and, therefore, parental transmission asymmetry would be observed at the next generation. A link between parental transmission asymmetry and penetrance of the mutation would thus be a strong argument in favor of a differential reproductive rate explaining parental transmission asymmetry. Indeed, such a correlation has never been described and will not be observed in the case of a parent-of-origin effect.

The goal of this paper is to test whether a differential reproductive rate explains the observed parental transmission asymmetry for RET CDS mutations, and more specifically to test if parental transmission asymmetry is correlated with penetrance of the mutation. In this aim, we take advantage of a unique worldwide sample of patients from the International Consortium on Hirschsprung Disease, gathering both sporadic cases and affected sib-pairs to study the correlation between parental transmission asymmetry and penetrance of the mutation. We also explore a possible complementary role of rs2435357 on parental transmission asymmetry.

\section{MATERIALS AND METHODS}

The data used in this paper have been obtained by the International Hirschsprung Disease Consortium. In this data set, CDS mutations have been searched for in 655 individuals and identified in 94 families, and among them were 21 affected sib-pairs and 73 sporadic cases (see Figure 1). Families from the US and Spanish data set have already been published. ${ }^{2,11,12}$

Because parental DNA was not available for all RET CDS mutation carriers patients, parental origin of RET CDS mutations was only searched for in 18 affected sib-pairs and 36 sporadic cases. A total of 15 de novo mutations were detected for sporadic cases. The parental origin of mutation was thus available for 21 sporadic cases. All information about these samples is summarized in Supplementary Table S1 and in Figure 1. Only two affected individuals had an affected parent (families 73 and 134, both from France).

Penetrances of the mutations are expected to be lower in sporadic cases than in sib-pairs. However, the classical approach of using the recurrence risk in the sibships of probands cannot be used as sibship sizes are not available in our sample and as most mutations are private. Consequently, to assess the fact that penetrances of the mutations are lower in sporadic cases than in affected sibpairs, we used the information on the mutation position. Mutations within the extracellular domain are considered to disrupt RET maturation/folding that subsequently fails to pass the endoplasmic reticulum quality control, ${ }^{13}$ leading to a more drastic loss of function than mutations lying in the transmembrane

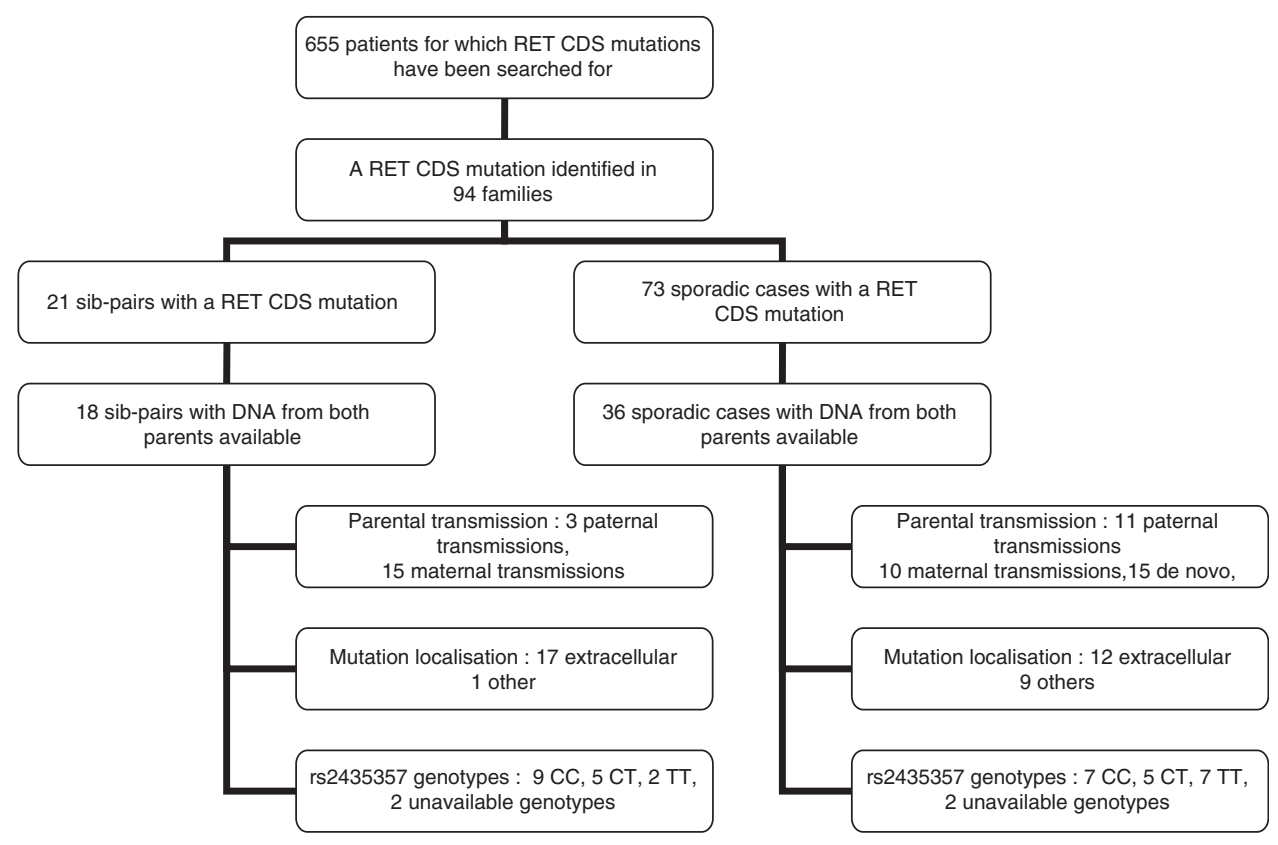

Figure 1 Diagram describing sample constitution of HSCR patients included in this study. 
and intracellular domain. Consequently, we considered that mutations within the extracellular domain have a higher penetrance than other mutations.

Thus, we tested, in a first step, whether the proportion of mutations within the extracellular domain was greater in affected sib-pairs than in sporadic cases and, in a second step, whether the parental transmission asymmetry was greater in affected sib-pairs and sporadic cases. Both tests were performed using the $\chi^{2}$ test.

As mutations penetrance was not complete (only two affected parents), we suspect that other factors on RET gene could modulate penetrance. We tested whether the risk allele frequency of rs2435357, known to have an increased frequency in RET mutation carrier HSCR patients, was more frequent in sporadic cases than in affected sib-pairs. Indeed, if rs2435357 risk allele frequency was decreased in affected sib-pairs compared with sporadic cases, this would be a further argument for penetrance of the mutation to be greater in affected sib-pairs than in sporadic cases. We also tested whether the risk allele in trans was more frequent in affected patients than in their unaffected parent in order to explain at least partly why most parents were unaffected.

All calculations have been performed using R software. ${ }^{14}$

\section{RESULTS}

There were 17 extracellular mutations versus only one other mutation for affected sib-pairs, whereas extracellular and other mutations had similar frequencies (12 extracellular versus 9 other mutations) for sporadic cases. The proportion of extracellular mutation was therefore significantly lower in sporadic and affected sib-pairs $(P=0.02)$, with sib-pairs having mutations with a higher penetrance.

Among sporadic cases, the RET CDS mutant allele was equally inherited from the mother and father (10 versus 11), whereas in affected sib-pairs there was a strong parental transmission asymmetry (15 from maternal origin versus 3 from paternal origin), giving a different parental transmission asymmetry among these samples $(P=0.05)$. Therefore, this positive association between penetrance of the mutation and parental transmission asymmetry allows us to conclude that the explanation for this asymmetry is that more severe mutations have resulted in a differential reproductive rate between male and female carriers.

We then tested the additional role of rs2435357 (cf, Supplementary Table S1). The difference of frequency for rs 2435357 risk allele between affected sib-pairs and sporadic cases was in the expected direction but only suggestive ( 9 out of 32 for sib-pairs versus 19 out of 38 for sporadic cases, $P=0.1$ ). We tested whether this polymorphism was more frequent in affected CDS mutation carriers than in their unaffected CDS mutation carrier parents. Interestingly, affected children carried the hypomorphic $\mathrm{T}$ allele in trans position more frequently than their unaffected CDS mutation carrier parents (12 out of 27 for children versus 3 out of 23 for carrier parents, $P=0.04$, see Supplementary Table S1).

\section{DISCUSSION}

We face two situations: sporadic cases for which no parental transmission asymmetry is observed and affected sib-pairs for which a strong parental transmission asymmetry is observed. This observation is positively correlated with the proportion of extracellular mutations in each sample. This result favors differential reproductive rates for male and female mutation carriers explaining the observed parental asymmetrical origin. We also underline here the complementary role of rs2435357 on RET CDS mutations with the risk allele in trans increasing penetrance. Therefore, the genotype of both parents affect RET CDS mutation penetrance in children, explaining why former segregation study results could not clearly distinguish between a dominant or recessive mode of inheritance. ${ }^{15}$

This study proposes to classify mutations using their localization. Indeed, a classification of mutations was not straightforward as mutations are private and only very few have been studied in vitro. ${ }^{13,16}$ Meanwhile, the classification that we propose here should not be used by clinicians as more specific classifications exist for some mutations, ${ }^{13,16}$ and this classification has no value for a particular mutation as some other features such as location on a binding site might increase strongly penetrance. For instance, one of the two paternally transmitted mutations in sibs in the extracellular domain was a missense change (p.Cys609Thr, family 88 from United States), known for its low penetrance. The other paternally transmitted mutation in sibs (p.Arg231His, family 49 from France) was a missense changing a basic histidine residue into another basic arginine residue in the extracellular domain, presumably of mild effect on the RET protein. Along the same lines, the only intracellular mutation found in a sib-pair (p.Met1064Thr, family 45 from France) affects codon 1064 close to the major phosphorylation site of the RET receptor (Tyr1062). Hence, that mutation, despite being located in the intracellular domain, may lead to a loss of function similar to the ones lying in the extracellular RET domain.

Other mechanisms commonly assumed when observing parental transmission asymmetry do not support a correlation between penetrance and parental transmission asymmetry or would involve mechanisms never described until yet to explain such correlation. If imprinting was involved, then we had to assume a differential imprinting among RET CDS mutations to explain such correlation. Such a situation has never been described in the literature. Anyway, imprinting is unlikely as the RET gene does not map to a known imprinted locus, although tissue-specific imprinting remains possible as demonstrated for the UBE3A $A^{17}$ or GNAS genes. ${ }^{18} \mathrm{~A}$ further attractive hypothesis was sex-specific genomic imprinting that would explain both the asymmetrical parental origin and the observed sex ratio. A sex-specific genomic imprinting has been found to be far more frequent than commonly assumed in mouse brain. ${ }^{19}$ However, this hypothesis was unlikely for HSCR as RET expression is biallelic in the gut. ${ }^{20}$ The question of a sperm-specific fitness explaining the parental transmission asymmetry, that is, a selective advantage for spermatozoids not carrying a RET CDS mutation, could also be raised, as the RET gene is known to play a crucial role in early spermatogenesis. $^{21}$ However, a selective advantage, ${ }^{22}$ and not disadvantage, has been described for spermatogonia carrying a RET mutation.

This study is a further argument against parental transmission asymmetry and the disease sex ratio in favor of females to be positively associated. Indeed, the sex ratio observed decreases with parental transmission asymmetry from 15:6 in sporadic case to 10:8 in affected sib-pairs. Therefore, the sex ratio decreases while parental transmission asymmetry increases. Furthermore, sex ratio decreases when the frequency of the RET CDS mutation increases; in a large sample of 882 probands with $13 \%$ of RET gene CDS mutations, ${ }^{5}$ the sex ratio was close to 5 whereas it dropped to 2 in our CDS mutation carriers sample. This prompts us to search for factor(s) other than RET gene responsible for the skewed sex ratio observed in HSCR.

It is likely that some genes involved in diseases have been suspected of a parent of origin, although sex-biased penetrance could also explain the observed data. Considering cleft lip with or without cleft palate, the sex ratio is biased with more affected males than females and an excess of maternal transmission in sporadic cases has been reported for several disease-causing genes. ${ }^{23-26}$ As cleft lip may harbor a social disadvantage and more males than females are affected, reduced fertility of affected individuals could lead to the observation of an excess of maternal transmission. Another example is hereditary cystatin C amyloid angiopathy (HCCAA), which is an autosomal dominant disease with high penetrance, responsible for brain 
hemorrhages in young normotensive adults. Intriguingly, the genealogies reveal that L68Q carriers experience a drastic reduction in lifespan from 65 years in the past to 30 years at present. ${ }^{27}$ During the same time period, a parental transmission asymmetry has emerged, whereby maternal inheritance of the mutation is associated with a 9-year reduction in lifespan relative to paternal inheritance. The reduction in lifespan combined with the emergence of an asymmetrical parental origin could be explained by the combination of a lower reproductive rate for patients and a higher disease penetrance in males compared with females. It could also explain why hereditary cerebral hemorrhage with amyloidosis-Dutch type (HCHWA-D) show an excess of mortality when the mutation is paternally inherited. ${ }^{28}$ Indeed, as this disease is more severe in females than in males, fewer female mutation carriers are likely to reach adulthood than males.

\section{CONFLICT OF INTEREST}

The authors declare no conflict of interest.

\section{ACKNOWLEDGEMENTS}

This study is a contribution from The International Hirschsprung Disease Consortium. We thank the numerous patients, families and referring physicians across the world who have participated in studies of Hirschsprung disease in our laboratories. This work was funded by the French National Research Agency (ANR, grants MRARE-HirGenet to SL, and ERare-HCR Consortium to SL, IC, and SB), the Fondation pour la Recherche Médicale (FRM) to SL and JA; the National Institutes of Health (R37 HD28088 to AC); the Italian Telethon (GGP04257 to IC); the Fondo de Investigación Sanitaria, Spain PI1001290 and Consejería de Economía, Innovación y Ciencia de la Junta de Andalucía (CTS7447) to SB; the NWO (901-04-225) Bernoulle Foundation and Ubbo Emmius Foundation to RMWH; and the Hong Kong Research Grant Council (HKU 765407 to MG-B and PKHT).

\section{WEB RESOURCES}

The URL for data presented herein is as follows: 1000 Genomes project, http://www.1000genomes.org/.

1 Bolk S, Pelet A, Hofstra RM et al: A human model for multigenic inheritance: phenotypic expression in Hirschsprung disease requires both the RET gene and a new 9q31 locus. Proc Natl Acad Sci USA 2000; 97: 268-273.

2 Gabriel SB, Salomon R, Pelet A et al: Segregation at three loci explains familial and population risk in Hirschsprung disease. Nat Genet 2002; 31: 89-93.

3 Amiel J, Sproat-Emison E, Garcia-Barcelo M et al: Hirschsprung disease, associated syndromes and genetics: a review. J Med Genet 2008; 45: 1-14.

4 Attie T, Pelet A, Edery $\mathrm{P}$ et al: Diversity of RET proto-oncogene mutations in familial and sporadic Hirschsprung disease. Hum Mol Genet 1995; 4: 1381-1386.

5 Emison ES, Garcia-Barcelo M, Grice EA et al: Differential contributions of rare and common, coding and noncoding Ret mutations to multifactorial Hirschsprung disease liability. Am J Hum Genet 2010; 87: 60-74.
6 Emison ES, McCallion AS, Kashuk CS et al: A common sex-dependent mutation in a RET enhancer underlies Hirschsprung disease risk. Nature 2005; 434: 857-863.

7 Becker T, Baur MP, Knapp M: Detection of parent-of-origin effects in nuclear families using haplotype analysis. Hum Hered 2006; 62: 64-76.

8 Haghighi F, Hodge SE: Likelihood formulation of parent-of-origin effects on segregation analysis, including ascertainment. Am J Hum Genet 2002; 70: 142-156.

9 Knapp M, Strauch K: Affected-sib-pair test for linkage based on constraints for identical-by-descent distributions corresponding to disease models with imprinting. Genet Epidemiol 2004; 26: 273-285.

10 Weinberg CR: Methods for detection of parent-of-origin effects in genetic studies of case-parents triads. Am J Hum Genet 1999; 65: 229-235.

11 Angrist M, Bolk S, Thiel B et al: Mutation analysis of the RET receptor tyrosine kinase in Hirschsprung disease. Hum Mol Genet 1995; 4: 821-830.

12 Ruiz-Ferrer M, Fernandez RM, Antinolo G, Lopez-Alonso M, Eng C, Borrego S: A complex additive model of inheritance for Hirschsprung disease is supported by both RET mutations and predisposing RET haplotypes. Genet Med 2006; 8: 704-710.

13 Kjaer S, Hanrahan S, Totty N, McDonald NQ: Mammal-restricted elements predispose human RET to folding impairment by HSCR mutations. Nat Struct Mol Biol 2010; 17: 726-731.

14 RD Core Team: R: A Language and Environment for Statistical Computing. R Foundation for Statistical Computing, Vienna, Austria, 2008.

15 Badner JA, Sieber WK, Garver KL, Chakravarti A: A genetic study of Hirschsprung disease. Am J Hum Genet 1990; 46: 568.

16 Kashuk CS, Stone EA, Grice EA et al: Phenotype-genotype correlation in Hirschsprung disease is illuminated by comparative analysis of the RET protein sequence. Proc Natl Acad Sci USA 2005; 102: 8949-8954.

17 Kishino T, Lalande M, Wagstaff J: UBE3A/E6-AP mutations cause Angelman syndrome. Nat Genet 1997; 15: 70-73.

$18 \mathrm{Yu}$ S, Yu D, Lee E et al: Variable and tissue-specific hormone resistance in heterotrimeric Gs protein alpha-subunit (Gsalpha) knockout mice is due to tissuespecific imprinting of the gsalpha gene. Proc Natl Acad Sci USA 1998; 95: 8715-8720.

19 Gregg C, Zhang J, Butler JE, Haig D, Dulac C: Sex-specific parent-of-origin allelic expression in the mouse brain. Science 2010; 329: 682-685.

20 Miao X, Leon TY, Ngan ES et al: Reduced RET expression in gut tissue of individuals carrying risk alleles of Hirschsprung's disease. Hum Mol Genet 2010; 19: 1461-1467

21 Jain S, Naughton CK, Yang M et al: Mice expressing a dominant-negative Ret mutation phenocopy human Hirschsprung disease and delineate a direct role of Ret in spermatogenesis. Development 2004; 131: 5503-5513.

22 Carlson KM, Bracamontes J, Jackson CE et al: Parent-of-origin effects in multiple endocrine neoplasia type 2B. Am J Hum Genet 1994; 55: 1076-1082.

23 Park BY, Sull JW, Park JY, Jee SH, Beaty TH: Differential parental transmission of markers in BCL3 among Korean cleft case-parent trios. J Prev Med Public Health 2009; 42: 1-4.

24 Sull JW, Liang KY, Hetmanski JB et al: Differential parental transmission of markers in RUNX2 among cleft case-parent trios from four populations. Genet Epidemiol 2008; 32: 505-512.

25 Sull JW, Liang KY, Hetmanski JB et al: Maternal transmission effects of the PAX genes among cleft case-parent trios from four populations. Eur J Hum Genet 2009; 17: 831-839.

26 Sull JW, Liang KY, Hetmanski JB et al: Evidence that TGFA influences risk to cleft lip with/without cleft palate through unconventional genetic mechanisms. Hum Genet 2009; 126: 385-394.

27 Palsdottir A, Helgason A, Palsson S et al: A drastic reduction in the life span of cystatin C L68Q carriers due to life-style changes during the last two centuries. PLoS Genet 2008; 4: e1000099.

28 Bornebroek M, Westendorp RG, Haan J et al: Mortality from hereditary cerebral haemorrhage with amyloidosis-Dutch type. The impact of sex, parental transmission and year of birth. Brain 1997; 120 (Part 12): 2243-2249.

Supplementary Information accompanies the paper on European Journal of Human Genetics website (http://www.nature.com/ejhg) 